\title{
Analyzing the temporal fluctuations of the reservoir-triggered seismicity observed at Açu (Brazil)
}

\author{
L. Telesca ${ }^{1}$, A. F. do Nascimento ${ }^{2}$, F. H. R. Bezerra ${ }^{3}$, and J. M. Ferreira ${ }^{2}$ \\ ${ }^{1}$ Istituto di Metodologie per l'Analisi Ambientale, CNR, C. da S. Loja, 85050 Tito (PZ), Italy \\ ${ }^{2}$ Departamento de Geofísica, Programa de Pós-graduação em Geodinâmica e Geofísica, Universidade Federal do Rio Grande \\ do Norte, Natal (RN), 59072-970, Brazil \\ ${ }^{3}$ Departamento de Geologia, Programa de Pós-graduação em Geodinâmica e Geofísica, Universidade Federal do Rio Grande \\ do Norte, Natal (RN), 59072-970, Brazil
}

Correspondence to: L. Telesca (luciano.telesca@imaa.cnr.it)

Received: 4 November 2011 - Revised: 20 December 2011 - Accepted: 21 December 2011 - Published: 28 March 2012

\begin{abstract}
The time dynamics of the reservoir-induced seismicity observed in Açu area (Brazil) from November 1994 to April 1997 reveals a super-Poissonian behaviour in the direction of a clustering process, where the occurrence of an earthquake increases the likelihood of the occurrence of a subsequent one. The seismicity shows strong correlation for time scales larger than approximately 1 day up to about four months, being characterized by Poissonian behavior for timescales smaller than 1 day. Processes of formation of fractures in the anisotropic and heterogeneous rockmass, along with pore pressure diffusion driven processes, are hypothesized as physical mechanisms for the appearance of Poissonian and clusterized dynamics respectively.
\end{abstract}

\section{Introduction}

The characterization of the temporal distribution of a seismic sequence represented a fundamental problem in seismology. This problem, in fact, is strictly connected to seismic hazard analysis (Boschi et al., 1995): knowing the statistical distribution ruling event occurrence improves the capability of reliably evaluating the probability of future earthquake occurrences. The models of time seismic occurrence used several distributions, among which the poissonian distribution was the most extensively used since in many cases, for large events a simple discrete Poisson distribution provides a close fit (Boschi et al., 1995). Poissonian processes are memoryless processes with all the events independent of each other, but it is well known that earthquake occurrence exhibits a strong degree of correlation between events of the same sequence. Such a property is also called timeclustering. Kagan and Jackson (1991) showed that earth- quakes are both short- and long-range time-clusterized; and the temporal clusterization of earthquakes was widely observed in many seismic catalogues (Smalley et al., 1987; Kagan and Jackson, 1991; Bodri, 1993; Telesca et al., 1999), with power-law interevent time distribution (Telesca et al., 2000a, b).

The interevent time distribution represents only one feature of a seismic process, because although it furnishes firstorder information about the process, it does not convey any information about correlation. Therefore, time dynamics of seismicity could be more deeply investigated by using second-order methods, like the fractal methods. These methods are linked with the power spectral density, which is the physical quantity that gives information about the correlation structures of a process. If the power spectrum is flat for any frequency, then the process is memoryless and uncorrelated, and this means that, for a point process like earthquakes, the time dynamics is Poissonian. Time-clusterized processes are, instead, characterized by a power spectrum behaving as a decreasing power-law (fractal) function of the frequency; and the power-law exponent, called scaling exponent, identifies and quantifies the strength of the inner time-correlations. Fractal methods were used to distinguish Poissonian from clusterized seismic sequences (Telesca et al., 2001a, b; Telesca et al., 2009), to evaluate the spatial variability of time-clustering behavior (Telesca et al., 2001c; Telesca et al., 2003), and to evaluate the magnitudevariability of the property of time-clusterization (Telesca and Macchiato, 2004; Telesca et al., 2007).

In the present study, time-clusterized structures in the time dynamics of the 1994-1997 Açu (Brazil) reservoirtriggered seismicity are analyzed. Reservoir-triggered seismicity represents one of the most interesting fields of the 
so-called anthropic seismicity and was observed in several areas worldwide, in Nurek Reservoir, Tadjikistan (Simpson and Negmatullaev, 1981), in the reservoirs LG3 and Manic 3, Quebec, Canada (Anglin and Buchbinder, 1985; Leblanc and Anglin, 1978), and in Koyna-Warna (India), which represented the paradigm of such seismic phenomenon (Telesca, 2010, and references therein). Reservoir-triggered earthquakes are characterized by higher $b$-value, larger magnitude ratio of the largest aftershock to the mainshock, increase of seismicity rate with the increase of water level; and these features discriminate them from tectonic seismic events (Gupta, 1985; Gupta et al., 1972a, b).

\section{Study area}

The study area lies on the Crystalline Precambrian (>540 Ma) basement of NE Brazil (Fig. 1). The area approaches the Cretaceous-Cenozoic sedimentary Potiguar basin. The crystalline basement is mostly composed of gneiss and migmatites (Caicó group) and mica-shists, marbles, and quartzites (Seridó group). Northeastern Brazil is a relatively stable intraplate area away from plate boundaries. Nonetheless, widespread evidence of coseismic faulting and liquefaction has been observed in the geological record (Bezerra et al., 2005, 2007). Historical data indicate that in 1808, an earthquake $4.8 \mathrm{mb}$ (estimated from felt area by Ferreira and Assumpção, 1983) had a maximum Modified Mercalli intensity of VI in the town of Açu. In 1930, another event was felt with intensity IV to V MM in Açu but the epicenter is not known. Additionally, swam-like seismic activity has been extensively reported and investigated in NE Brazil using local seismographic networks (Ferreira et al., 1998, 2008; Lopes et al., 2010; Bezerra et al., 2007).

The crystalline basement in NE Brazil provides excellent signal-to-noise ratios in the recorded waveforms due to its high rigidity, simple seismic velocity structure, and low attenuation. This characteristic, along with its relatively low background seismicity, makes the region an excellent site for studies concerning the following: shear-wave splitting (do Nascimento et al., 2004a), spatio-temporal evolution of reservoir-induced seismicity (Ferreira et al., 1995; do Nascimento et al., 2004b; El-Hariri et al., 2010), seismic sources (Tomic et al., 2009) and fault/fracture transport properties (do Nascimento et al., 2005a, b; Pytharouli et al., 2011).

Seismicity in the Açu reservoir occurs in NE-SW trending discrete fault zones (Ferreira et al., 1995; do Nascimento et al., 2004a), although more detailed work using field observation and high precision hypocentral location has demonstrated that the earthquakes are not occurring in a single plane defined by the hypocentral location (Pytharouli et al., 2011). In fact, the seismicity occurs in a series of sub parallel and perpendicular planes to the NE-SW trend. Nonetheless, all these planes are consistent with the modern-day E-W compressive stress (Pytharouli et al., 2011).

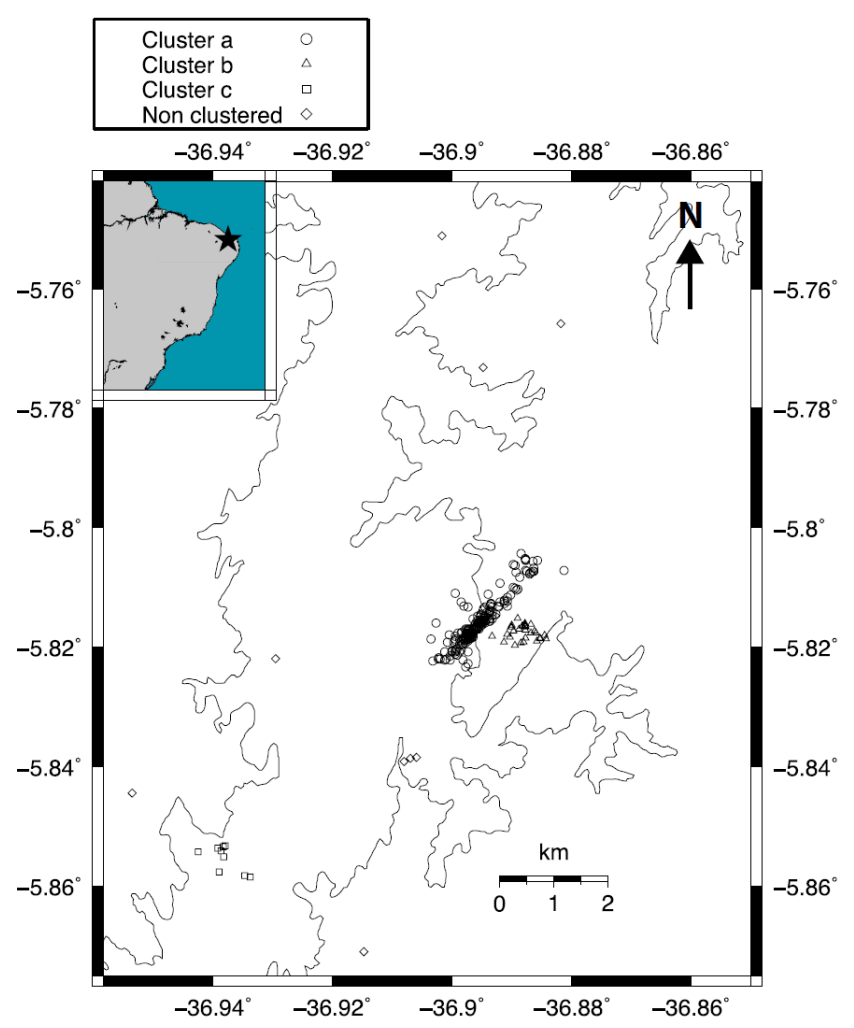

Fig. 1. Spatial distribution of the seismic clusters $a, b$ and $c$ in Açu (NE Brazil).

The report by do Nascimento et al. (2004a) reported the occurrence of at least four discrete active fault zones - they called them "clusters" - in the Açu reservoir region. For three of these clusters (clusters a, b and c in Fig. 1), it was possible to capture their detailed spatio-temporal behavior with respect to the water level in the reservoir. Unfortunately, only during the mentioned three-year period we had proper monitoring using a eight three-component digital network. After that period, the project that funded the seismic monitoring ended and so did the monitoring using such dense network. After 1997, we only kept one analogue station near the dam wall for a few more months. The relationship between average time delay between the maximum in water level and the average hypocentral depth of the seismic activity revealed that clusters $a$ and $b$ have slightly lower hydraulic conductivity than cluster $\mathrm{c}$, which reflects the heterogenous nature of the fault permeability structures (do Nascimento et al., 2004a).

In fact, pore pressure diffusion is the dominant RIS mechanism at the site. However, other mechanims also play a role at the site. Certainly undrained response is a possible explanation for seismic triggering in 1994, so that seismicity increased shortly after the increase in water level. The higher seismicity rate in 1995 is probably due to the initiation of the seismicity in "cluster a" and additional seismicity 


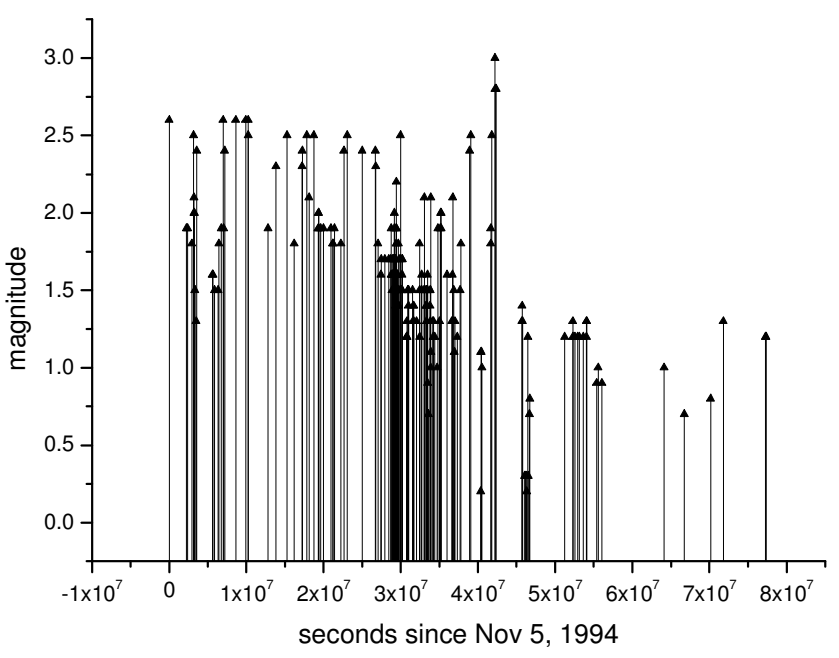

Fig. 2. Time distribution of the earthquakes of cluster a. The number of events is 224 .

some other area in the reservoir. At the water-level peak in 1995 , the reservoir was $32.8 \mathrm{~m}$ deep, and the seismicity rate was significantly higher following this larger peak, the highest the water-level had been since 1989. The observation of this high seismicity rate after the highest water level in the reservoir since 1989 could also be a manifestation of the Kaiser effect (similarly to the observations of Durá-Gómez and Talwani, 2009), so that we could observe the occurrence of large magnitude events when the lake level exceeds the previous maximum lake-level. However, to address this issue we would have to investigate how the magnitudes are related to the seismicity and water level, which is beyond the scope of the present work.

\section{Methods and data analysis}

The temporal distribution for cluster a is shown in Fig. 2. Cluster $\mathrm{b}$ and $\mathrm{c}$ were not investigated due to their very low number of events. The sequence is visibly clusterized in time because the events are not homogeneously distributed on time.

Figure 3 shows the typical cumulative number of events $N(t)$ versus time $t$. One observes that the local rate (local slope) is not constant, contrary to a Poisson process; in fact, it is clearly visible that a jump-like feature located approximately in the middle of the series is superimposed to an irregular trend.

In order to depict more precisely rate inhomogeneities, an analysis of the local rates was performed. The whole observation period was subdivided in a certain number of nonoverlapping windows $W$ of duration $T$ and the number of events $N_{\mathrm{w}}$ falling in each window was counted. The local rate $R_{\mathrm{w}}$ was then given by $N_{\mathrm{w}} / T$. Figure 4 shows, as an example, the

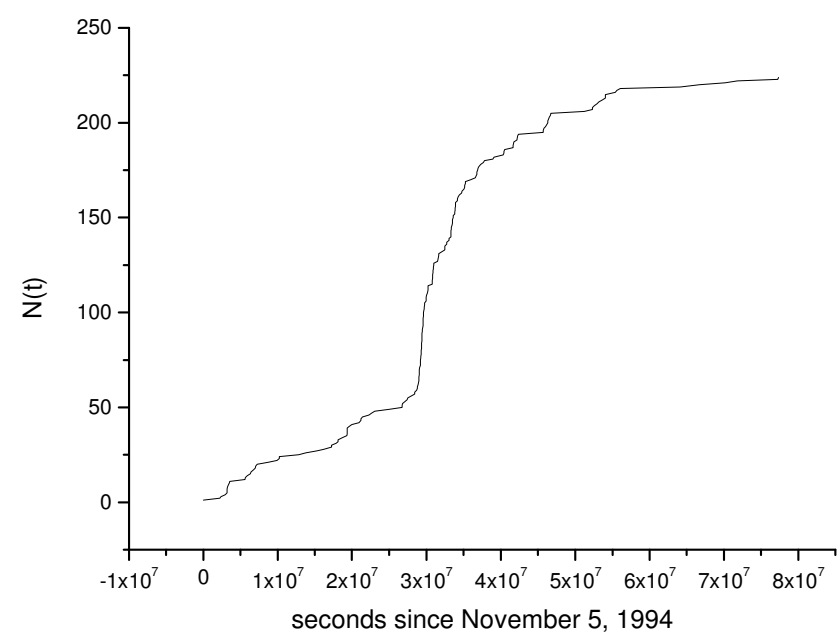

Fig. 3. Cumulative number of events $N(t)$ vs. time of occurrence for cluster a.

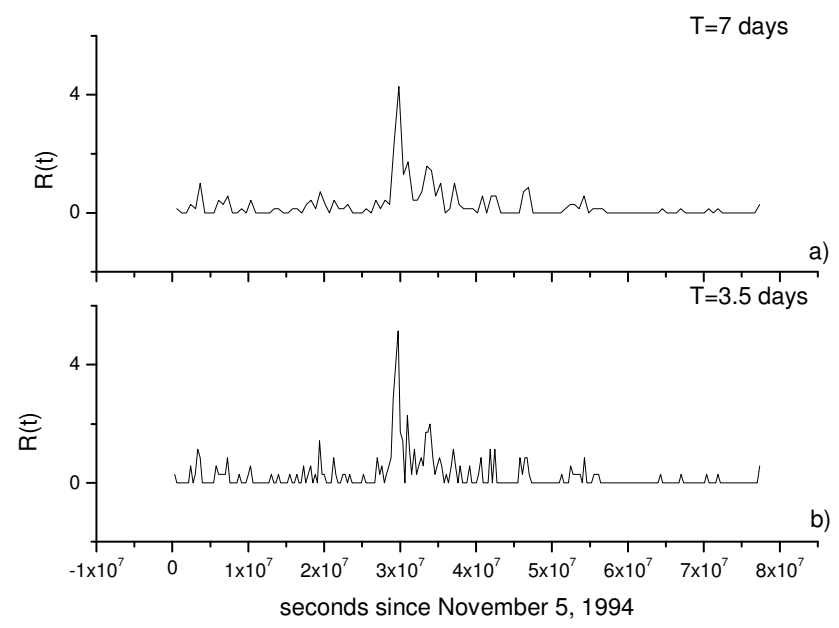

Fig. 4. Local rates for cluster a with time window of 7 days (a) and 3.5 days (b).

local rate $R_{\mathrm{W}}$ for $T=7$ days (Fig. $4 \mathrm{a}$ ) and for $T=3.5$ days (Fig. 4b). In both cases, a quasi-monthly oscillation of the rate is visible. To estimate rate's linear correlations, a spectral analysis of the time series of increments (as difference between consecutive values) of the rate $R_{\mathrm{w}}$ was performed. Figure 5 shows the log-log plot of the power spectral density $S(f)$ of the increment time series for the rate $R_{\mathrm{w}}$ shown in Fig. 4a. The spectrum is approximately linear, following the power law $S(f) \sim f^{\beta}$, with $\beta \sim 0.7$. The particular shape of the spectrum, increasing with a power-law function of the frequency $f$, indicates that the consecutive values of the increments $I$ are negatively correlated or antipersistent, meaning that the increase in the rate is very likely followed by a decrease and vice versa. The shuffled increment time series is, instead, a white noise (Fig. 6). Since the increment 


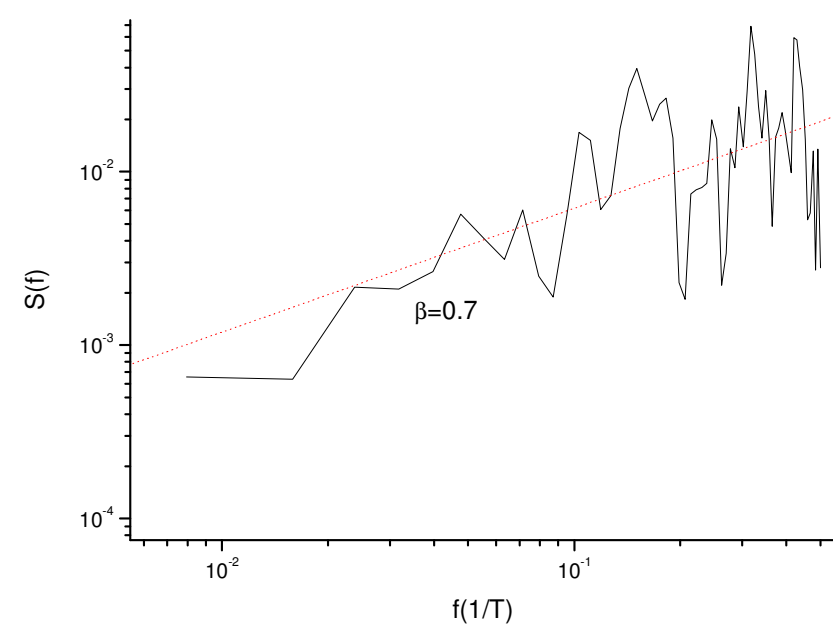

Fig. 5. Power spectrum of the increment time series for the rate shown in Fig. 4a.

process $I$ is the derivative of the rate process $R_{\mathrm{w}}$, the spectrum of $R_{\mathrm{W}}$ decreases as a power-law function $S(f) \sim 1 / f^{\alpha}$, with $\alpha=2-\beta$ (Peng et al., 1993).

The Allan Factor (AF) is applied to detect correlations in the sequence of the earthquake counts. Dividing the time axis into equally spaced contiguous windows of duration $\tau$, and denoting with $N_{k}(\tau)$ the number of events falling into the $k$-th window, the Allan Factor is defined as $\operatorname{AF}(\tau)=\frac{<\left(N_{k+1}(\tau)-N_{k}(\tau)\right)^{2}>}{2<N_{k}(\tau)>}$, where $<\ldots>$ indicates expectation value.

If the sequence of earthquakes is clusterized in the time domain, then $\operatorname{AF}(\tau)$ behaves as a power-law function, $\operatorname{AF}(\tau) \propto$ $\tau^{d}$ (Thurner et al., 1997), and the fractal exponent $d$ can be estimated by the slope of the line that fits the curve in its linear range; for a hypothetical Poissonian earthquake sequence, the AF is approximately near unity for all timescales $\tau$, with $\alpha \approx 0$.

Figure 7 shows the $\mathrm{AF}$ of the seismic sequence recorded in Açu area for timescales $\tau$ from $10 \mathrm{~s}$ to about 3 months; the upper timescale approximately corresponds to the $1 / 10$ of the entire period, as higher timescales would lead to misleading results for the poorer statistics. The AF plot suggests the presence of time-clustering behavior because it increases with linear form for $\tau>10^{5} \mathrm{~s}$ in bilogarithmic scales. The cutoff timescale $10^{5} \mathrm{~s}$ is the so-called fractal onset time (Thurner et al., 1997) and indicates the lower timescale from which the clustering behavior can be detected and quantified. The early flatness up to about $10^{4} \mathrm{~s}$ indicates a Poissonianlike behavior of the sequence for the small timescales. The intermediate timescale region between $10^{4} \mathrm{~s}$ and $10^{5} \mathrm{~s}$ can be considered as a "transfer" timescale region between the two opposite behaviors - from Poissonian to clusterized dynamics. The estimate of the scaling exponent in such timescale range is $\sim 0.7$. The $\mathrm{AF}$ curve shows a drop at around

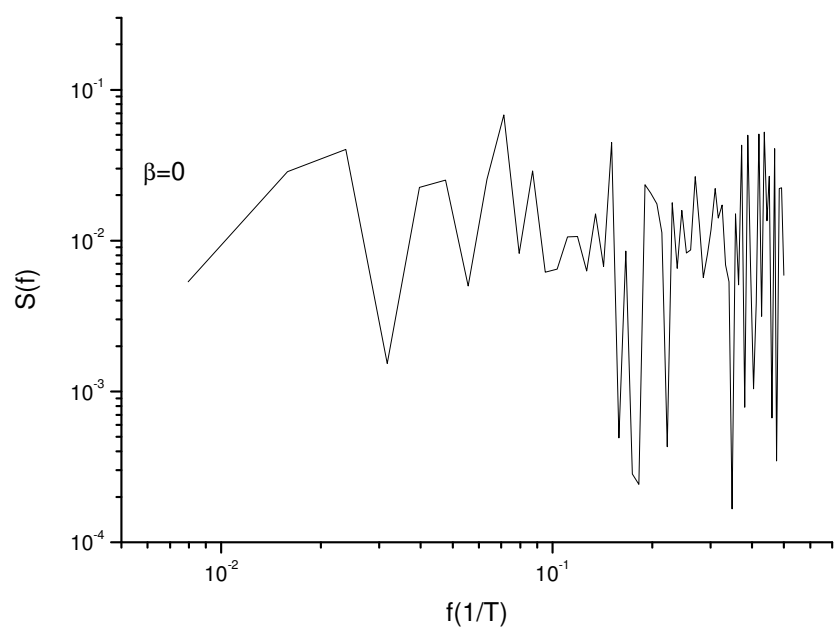

Fig. 6. Power spectrum of the shuffled increment time series.

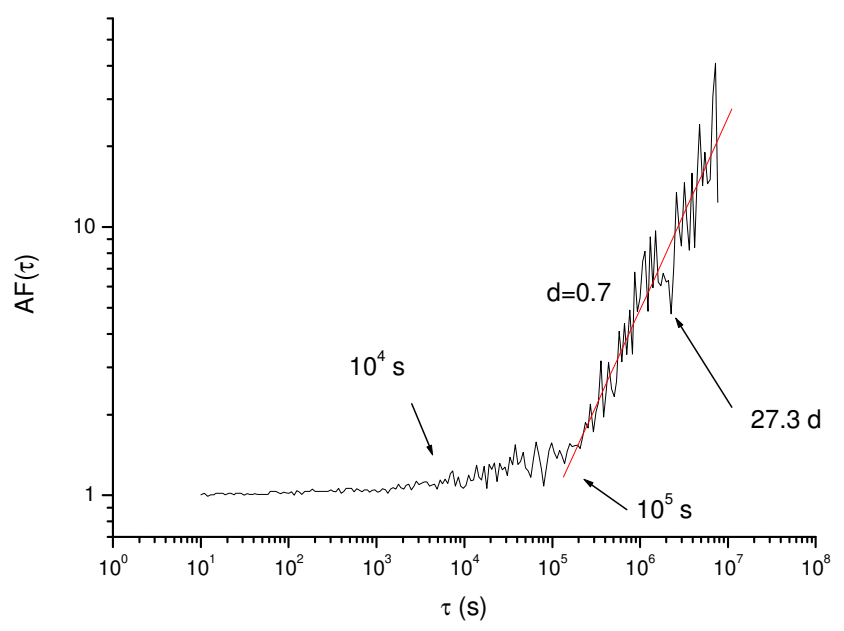

Fig. 7. Allan Factor of the seismic series of cluster a.

27.3 days; generally a drop in the AF curve indicates a periodicity in the sequence (Turcott et al., 1994).

In order to check whether the AF curve is significantly distinguished from that obtained by Poissonian sequences, characterized by identical mean intervent time and identical number of events, we generated 1000 Poissonian sequences. To each simulated sequence the AF was applied. For each timescale the 95th percentile among the AF values for that timescale was calculated. The final $95 \%$ confidence AF curve was then given by the set of the 95th percentiles. The AF curve is significantly different from those obtained by the Poisson surrogates for $\tau>10^{4} \mathrm{~s}$; therefore, the scaling behavior of the seismic cluster is significantly non Poissonian (Fig. 8). It is clear that for timescales shorter than $10^{3} \mathrm{~s}$ the point process of earthquakes is essentially Poissonian, because the AF remains close to unity. For longer timescales 


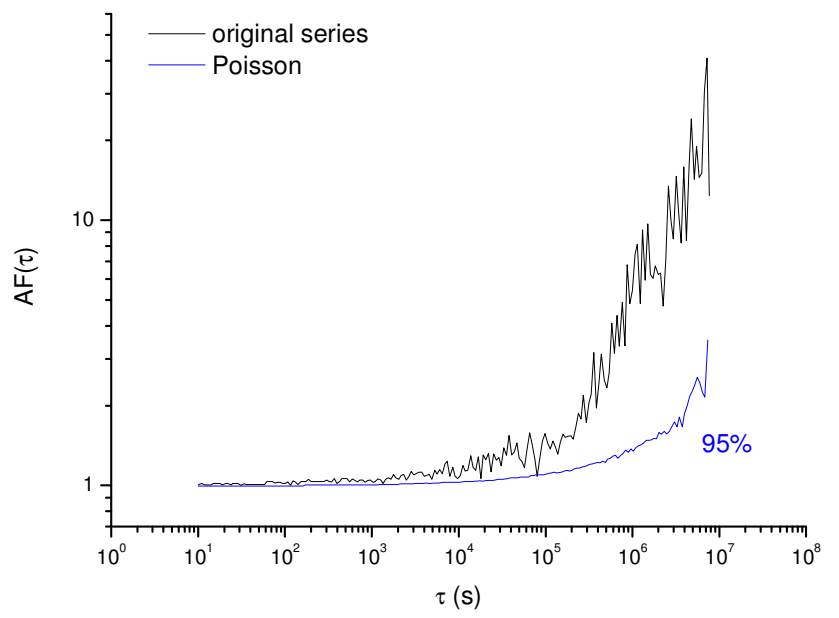

Fig. 8. Comparison between the AF of the cluster a and the $95 \%$ confidence curve evaluated over 1000 Poisson surrogates.

the AF curve noticeably departs from unity, suggesting that there might be some non-Poissonian effects in the seismic process.

In order to check the possible origin of the scaling behavior of the sequence, we shuffled 1000 times the interevent intervals, and for each shuffle we calculated the AF curve. The $95 \%$ confidence AF curve for the shuffles was calculated as above (Fig. 9). If the original point process exhibits the same behavior in AF as the shuffled one, then the observed departure from Poissonian statistics is only due to the distribution of interevent intervals and not due to their particular ordering; that is, the interevent intervals are no more bursty than expected by chance (Anteneodo et al., 2010). The shuffling reduces mostly the upper bound of the power scaling region from the timescale $\tau>10^{6} \mathrm{~s}$, and this indicates that the scaling properties are partially due to the distribution of the interevent times, but also that the longer range correlations are due to the specific ordering of the interevent intervals. In particular, up to $10^{6} \mathrm{~s}$ the AF curve is not discernably different from the shuffled counterpart (with $95 \%$ confidence), indicating that the observed "departure" from Poissonian statistics (for timescales less than $10^{6} \mathrm{~s}$ ) is merely an artifact of the heavy-tailed distribution of the interevent intervals and not due to some intrinsic burstiness. On the other hand, for timescales higher than $10^{6} \mathrm{~s}$, the $\mathrm{AF}$ of the original sequence is above that of the shuffle, and this indicates that for these timescales the dynamics of the seismic process is due to the ordering of the interevent intervals.

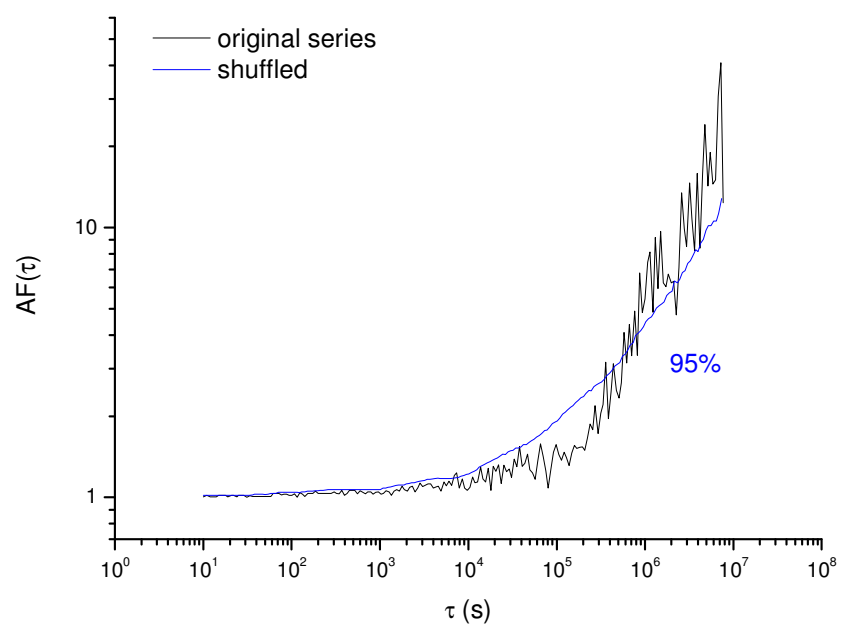

Fig. 9. Comparison between the $\mathrm{AF}$ of the cluster a and the $95 \%$ confidence curve evaluated over 1000 shuffled surrogates.

\section{Discussion and conclusions}

Time-scaling dynamical analysis of seismicity data observed in Açu (Brazil) reservoir from November 1994 to April 1997 in the temporal dynamics of the seismic series in the spatial "cluster a" reveals super-Poissonian behaviour in the direction of a clustering process, where the occurrence of an earthquake increases the likelihood of the occurrence of a subsequent one.

The seismicity of this cluster in the study area is known to be strongly associated with the pore diffusion through fractures imbedded in the rockmass.

The cluster shows strong correlation for time scales $\tau>10^{5} \mathrm{~s}$ (approximately 1 day) reaching up to $10^{7} \mathrm{~s}$ (approximately four months). For timescale $\tau<10^{4} \mathrm{~s}$ (hour scale) the interevent time distribution is Poissonian. This is consistent with the dominance in seismicity by the widespread formation of fractures in the anisotropic and heterogeneous rockmass at short timescales. For timescales larger than $\tau>10^{6} \mathrm{~s}$ (day to month scale), for which the clustering is mainly due to the ordering of the interevent times, the seismicity is mainly driven by a pore pressure diffusion mechanism which has similar timescale characteristics (days to months). The reason for the observed periodicity of about 27 days, indicated by the drop in AF curve, is not well-know; probably it could be connected with Earth tidal processes. Klein (1976) found that six over eight reservoirinduced seismic sequences revealed tidal components, identifying the role of tidal stresses in being oriented to enhance the tectonic stress. However, in the case examined in our paper, deeper investigation will be planned in order to assess the existence of tidal components in the time evolution of seismicity. 
Acknowledgements. L. Telesca acknowledges the financial support of CNR in the framework of the Short-term mobility Program 2010. A. do Nascimento, J. Ferreira and F. Bezerra thank CNPq for their PQ grants and also the National Institute of Science and Technology $(\mathrm{CNPq}$ - INCT-Estudos Tectônicos and INCT-Geofísica do Petróleo). A. do Nascimento also thanks $\mathrm{CNPq}$ for grant Proc. 483349/2007-0. Figure 1 was made using GMT (Generic Mapping Tools) from Wessel and Smith (1991).

Edited by: M. E. Contadakis

Reviewed by: G. Babayev and another anonymous referee

\section{References}

Anglin, F. M. and Buchbinder, G. G. R.: Induced seismicity at the LG3 Reservoir, James Bay, Quebec, Canada, Bull. Seismol. Soc. USA, 75, 1067-1076, 1985.

Anteneodo, C., Malmgren, R. D., and Chialvo, D. R.: Poissonian bursts in e-mail correspondence, Eur. Phys. J. B, 75, 389-394, 2010.

Bezerra, F. H. R., Fonseca, V. P., Vita-Finzi, C., Lima-Filho, F. P., and Saadi, A.: Liquefaction-induced structures in Quaternary alluvial gravels and gravels sediments, NE Brazil, in: Paleoliquefaction and Appraisal of Seismic Hazards, edited by: Obermeier, S. F., Eng. Geol., 76, 191-208, 2005.

Bezerra, F. H. R., Takeya, M. K., Sousa, M. O. L., and do Nascimento, A. F.: Coseismic reactivation of the Samambaia fault, Tectonophysics, 430, 27-39, 2007.

Bodri, B.: A fractal model for seismicity at Izu-Tokai region, Central Japan, Fractals, 1, 539-546, 1993.

Boschi, E., Gasperini, P., and Mulargia, F.: Forecasting where larger crustal earthquakes are likely to occur in Italy in the near future, Bull. Seism. Soc. Am., 85, 1475-1482, 1995.

do Nascimento, A. F., Cowie, P. A., Lunn, R. J., and Pearce, R. G.: Spatio- temporal evolution of induced seismicity at Açu reservoir, NE Brazil, Geophys. J. Int., 158, 1041-1052, 2004a.

do Nascimento, A. F., Bezerra, F. H. R., and Takeya, M. K.: Ductile Precambrian fabric control of seismic anisotropy in the Açu dam area, northeastern Brazil, J. Geophys. Res., 109, B10311, doi:10.1029/2004JB003120, 2004b.

do Nascimento, A. F., Lunn, R. J., and Cowie, P. A.: Numerical modelling of pore-pressure diffusion in a reservoir-induced seismicity site in northeast Brazil, Geophys. J. Int., 160, 249-262, 2005a.

do Nascimento, A. F., Lunn, R. J., and Cowie, P. A.: Modeling the heterogeneous hydraulic properties of faults using constraints from reservoir-induced seismicity, J. Geophys. Res., 110, B09201, doi:10.1029/2004JB003398 2005b.

Durá-Gómez, I. and Talwani, P.: Hydromechanics of the KoynaWarna Region, India. Pure Appl. Geophys., 167, 183-213, 2009.

El Hariri, M., Abercrombie, R. E., Rowe, C. A., and do Nascimento, A. F.: The role of fluids in triggering earthquakes: observations from reservoir induced seismicity in Brazil. Geophys. J. Int., 181, 1566-1574, 2010.

Ferreira, J. M. and Assumpção, M.: Sismicidade do Nordeste do Brasil, Rev. Bras. Geofis., 1, 67-88, 1983.

Ferreira, J. M., Oliveira, R. T., Assumpcão, M., Moreira, J. A. M., Pearce, R. G., and Takeya, M. K.: Correlation of seismicity and water level in the Açu reservoir-an example from Northeast Brazil, Bull. Seismol. Soc. Am., 85, 1483-1489, 1995.

Ferreira, J. M., Oliveira, R. T., Takeya, M. K., and Assumpção, M.: Superposition of local and regional stresses in Northeast Brazil: evidence from focal mechanisms around the Potiguar marginal basin, Geophys. J. Int., 134, 341-355, 1998.

Ferreira, J. M., Bezerra, F. H. R., Sousa, M. O. L., do Nascimento, A. F., Sá, J. M., and França, G. S.: The role of Precambrian mylonitic belts and present-day stress field in the coseismic reactivation of the Pernambuco lineament, Brazil, Tectonophysics, 456, 111-126, 2008.

Gupta, H. K.: The present status of reservoir indiced seismicity investigations with special emphasis on Koyna earthquakes, Tectonophysics, 118, 257-279, 1985.

Gupta, H. K., Rastogi, B. K., and Narain, H.: Common features of the reservoir-associated seismic activities, Bull. Seismol. Soc. Am., 62, 481-492, 1972a.

Gupta, H. K., Rastogi, B. K., and Narain, H.: Some discriminatory characteristics of earthquakes near the Kariba, Kremasta, and Koyna artificial lakes, Bull. Seismol. Soc. Am., 62, 493-507, 1972 b.

Kagan, Y. Y. and Jackson, D. D.: Long-term earthquake clustering, Geophys. J. Int., 104, 117-133, 1991.

Klein, F. W.: Tidal triggering of reservoir-associated earthquakes, Eng. Geol., 10, 197-210, 1976.

Leblanc, G. and Anglin, F.: Induced seismicity at the Manic 3 Reservoir, Quebec, Bull. Seismol. Soc. Am., 68, 1469-1485, 1978.

Lopes, A. E. V., Assumpção, M., do Nascimento, A. F., Ferreira, J. M., Menezes, E. A. S., and Barbosa, J. R.: Intraplate earthquake swarm in Belo Jardim, NE Brazil: reactivation of a major Neoproterozoic shear zone (Pernambuco Lineament), Geophys J. Int., 180, 1303-1312, 2010.

Peng, C.-K., Mietus, J., Hausdorff, J. M., Havlin, S., Stanley, H. E., and Goldberger, A. L.: Long-range anticorrelations and nonGaussian behavior of the heartbeat, Phys. Rev. Lett., 70, 1343 1346, 1993.

Pytharouli, S. I., Lunn, R. J., Shipton, Z. K., Kirkpatrick, J. D., and do Nascimento, A. F.: Microseismicity illuminates open fractures in the shallow crust, Geophys. Res. Lett., 38, L02402, doi:10.1029/2010GL045875, 2011.

Simpson, D. W. and Negmatullaev, S. K.: Induced seismicity at Nurek Reservoir, Tadjikistan, USSR, Bull. Seismol. Soc. Am., 71, 1561-1586, 1981.

Smalley, R. F., Chatelain, J.-L., Turcotte, D. L., and Prévot, R.: A fractal approach to the clustering of earthquakes: applications to the seismicity of the New Hebrides, Bull. Seismol. Soc. Am., 77, 1368-1381, 1987.

Telesca, L.: Analysis of the cross-correlation between seismicity and water level in Koyna area (India), Bull. Seismol. Soc. Am., 100, 2317-2321, 2010.

Telesca, L. and Lovallo, M.: Non-uniform scaling features in central Italy seismicity: a non-linear approach in investigating seismic patterns and detection of possible earthquake precursors, Geophys. Res. Lett., 36, L01308, doi:10.1029/2008GL036247, 2009.

Telesca, L. and Macchiato, M.: Time-scaling properties of the Umbria-Marche 1997-1998 seismic crisis, investigated by the Detrended Fluctuation Analysis, Chaos Solit. Fract., 19, 377- 
385, 2004.

Telesca, L., Cuomo, V., Lanfredi, M., Lapenna, V., and Macchiato, M.: Investigating Clustering Structures in Time-Occurrence Sequences of Seismic Events Observed in the Irpinia- Basilicata Region (Southern Italy), Fractals, 7, 221-234, 1999.

Telesca, L., Cuomo, V., Lapenna, V., and Vallianatos, F.: Selfsimilarity properties of seismicity in the Southern Aegean area, Tectonophysics, 321, 179-188, 2000a.

Telesca, L., Cuomo, V., Lapenna, V., and Macchiato, M.: Analysis of the time-scaling behaviour in the sequence of the aftershocks of the Bovec (Slovenia) April 12, 1998 earthquake, Phys. Earth Planet. Int., 120, 315-326, 2000 b.

Telesca, L., Cuomo, V., Lapenna, V., and Macchiato, M.: Statistical analysis of fractal properties of point processes modelling seismic sequences, Phys. Earth Planet. Int., 125, 65-83, 2001a.

Telesca, L., Cuomo, V., Lapenna, V., and Macchiato, M.: Intermittent-type temporal fluctuations in seismicity of the Irpinia (southern Italy) region, Geophys. Res. Lett., 28, 37653768, 2001b.

Telesca, L., Cuomo, V., Lapenna, V., and Macchiato, M.: Depthdependent time-clustering behavior in seismicity of southern California, Geophys. Res. Lett., 28, 4323-4326, 2001c.
Telesca, L., Lapenna, V., and Macchiato, M.: Spatial variability of time-correlated behaviour in Italian seismicity, Earth Planet. Sci. Lett., 212, 279-290, 2003.

Telesca, L., Lovallo, M., Lapenna, V., and Macchiato, M.: Longrange correlations in 2-dimensional spatio-temporal seismic fluctuations, Phys. A, 377, 279-284, 2007.

Thurner, S., Lowen, S. B., Feurstein, M. C., Heneghan, C., Feichtinger, H. G., and Teich, M. C.: Analysis, Synthesis, and Estimation of Fractal-Rate Stochastic Point Processes, Fractals, 5, 565-596, 1997.

Tomic, J., Abercrombie, R. E., and do Nascimento, A. F.: Source parameters and rupture velocity of small $<2.1$ reservoir induced earthquakes, Geophys. J. Int., 179, 1013-1023, 2009.

Turcott, R. G., Lowen, S. B., Li, E., Johnson, D. H., Tsuchitani, C., and Teich, M. C.: A Nonstationary Poisson Point Process Describes the Sequence of Action Potentials Over Long Time Scales in Lateral-Superior-Olive Auditory Neurons, Biol. Cybern., 70, 209-217, 1994.

Wessel, P. and Smith, W. H. F.: Free software helps map and display data, EOS Trans. AGU, 72, 441, 1991. 